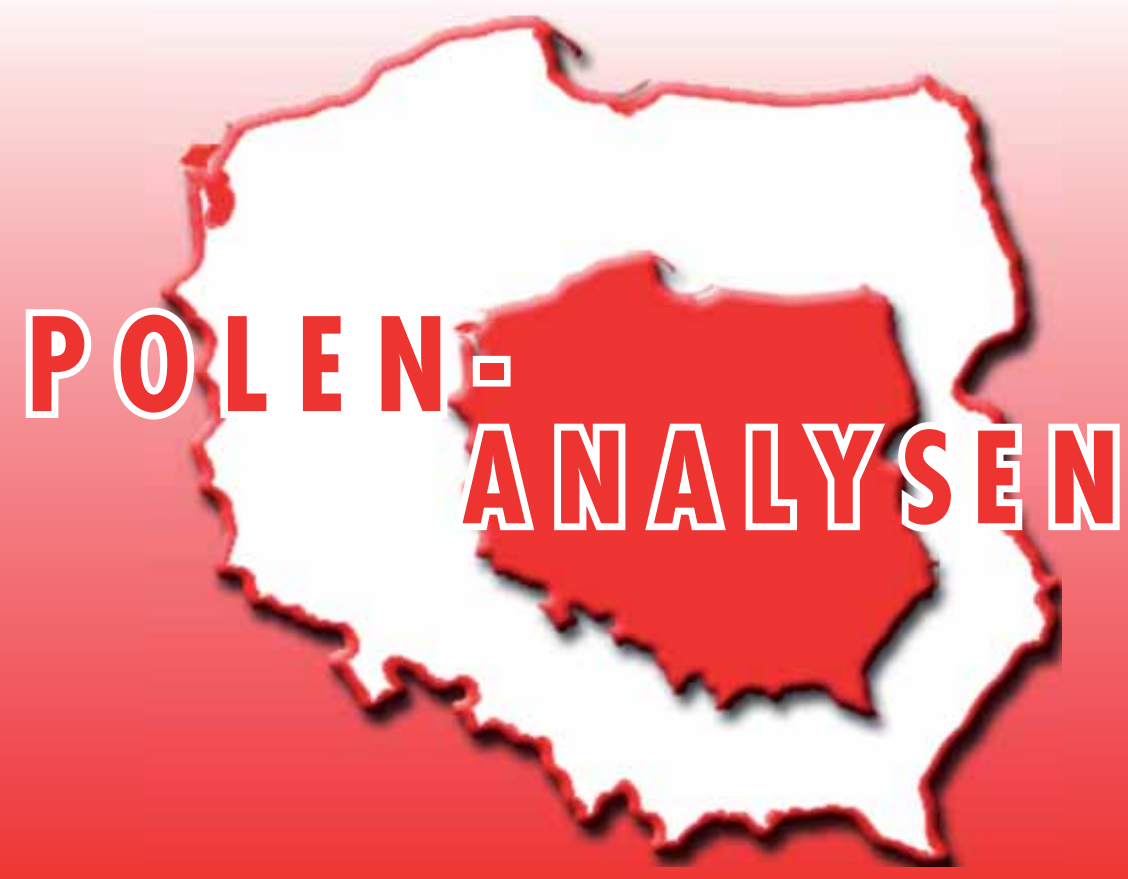

www.laender-analysen.de/polen

\title{
NEUERE ENTWICKLUNGEN IM HOCHSCHULSEKTOR
}

ANALYSE

Neuere Entwicklungen im Hochschulsektor

Sonja Steier, Bochum

Die Abschaffung der Habilitation in Polen in der Diskussion

Fryderyk Zoll, Krakau

TABELLEN UND GRAFIKEN

Das polnische Hochschulsystem

CHRONIK

Vom 18. März bis zum 31. März 2008

\section{- Forschungsstelle Osteuropa}

Deutsche

Gesellschaft für Osteuropakunde e.V. 


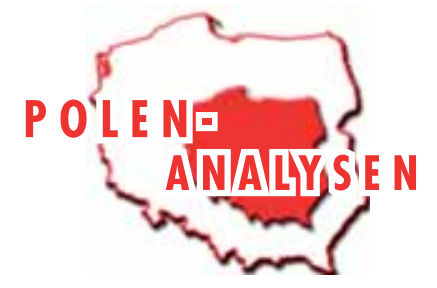

Analyse

\section{Neuere Entwicklungen im Hochschulsektor}

Die Wiederherstellung der Hochschulautonomie sowie die Aufwertung der Hochschulen gegenüber der Polnischen Akademie der Wissenschaften mit einhergehender Studienreform gehörten zu den bedeutendsten Reformschritten zu Beginn der postkommunistischen Ära, wie Sonja Steier darstellt. Für die Hochschulen bedeutete die neue Hochschulpolitik auch eine ausgeprägte Differenzierung und Konkurrenz untereinander. Zugleich kam es zu einer Gründungswelle nichtstaatlicher Hochschulen unterschiedlichster Qualität. Infolge einer in den 1990er Jahren einsetzenden Bildungsexpansion konnten die Hochschulen die Studierendenzahlen innerhalb weniger Jahre vervierfachen; die Zahl der Hochschulen stieg ebenfalls um ungefähr denselben Faktor. Seit 1999 wird die Entwicklung des polnischen Hochschulsystems auch vom Bologna-Prozess gesteuert, der zu einer neuen Diversifizierung der Studiengangstrukturen führt.

Im Rahmen der seit Jahren andauernden Reformdiskussionen im Hochschulbereich hat vor kurzem eine vom Wissenschafts- und Hochschulministerium eingesetzte Arbeitsgruppe die Grundzüge einer Reform vorgelegt, deren Kern die Abschaffung der Habilitation sein soll, mit dem Ziel, die wissenschaftliche und berufliche Laufbahn von jungen Wissenschaftlern zu erleichtern. Die Abschaffung der Habilitation beurteilt Fryderyk Zoll allerdings nicht als Heilmittel, da damit nicht automatisch neue Anreize zur Mobilität der Wissenschaft geschaffen würden. Den Ansatz für eine Innovation sieht er vielmehr in der Etablierung eines durchsichtigen Auswahlprozesses bei der Besetzung von Professorenstellen, verbunden mit einer besseren Bezahlung, die die Motivation für einen vollen Einsatz an der Hochschule entscheidend erhöhen und die qualitätssenkende berufliche Mehrfachbelastung von Professoren zurückdrängen soll.

\section{Neuere Entwicklungen im Hochschulsektor}

Sonja Steier, Bochum

\section{Bildungspolitische Neuorientierung}

Die Verhandlungen am Runden Tisch im Frühjahr 1989, die u.a. auch eine Debatte über die Richtung der polnischen Bildungspolitik und die künftige Gestalt des Bildungssystems zum Thema hatten, leiteten den generellen Systemwechsel in Polen ein. Damit vollzog sich auch die Ablösung von den ideologischen Leitwerten, den Strukturen und Praxiswerten des staatssozialistischen Bildungssystems.

Die Wiederherstellung der Hochschulautonomie mit der Verankerung von Forschung und Lehre sowie die Aufwertung der Hochschulen gegenüber der Polnischen Akademie der Wissenschaften bei weit reichender Freiheit mit Blick auf die Studienreform (Studienstrukturen und -gänge) gehörten zu den bedeutendsten Reformschritten zu Beginn der postkommunistischen Ära. Für die Hochschulen bedeutete es neben der relativen Unabhängigkeit vom Staat (Steuerung, Gebührenfreiheit) und der institutionellen Autonomie auch eine ausgeprägte Differenzierung und Konkurrenz der Hochschulen (Rankinglisten) untereinander. Die bekanntesten und renommiertesten Hochschulen sind die Universitäten Warschau, Krakau, Breslau und Posen. Kattowitz, Lublin, Lodz und Danzig zählen ebenfalls zu den großen Wissenschaftszentren des Landes.
Zugleich zeigt die Gründungswelle der nichtstaatlichen Hochschulen eine ungewöhnliche und anhaltende Entwicklungsdynamik. Im Studienjahr 1991/92 gab es erst zwölf solcher Einrichtungen, dagegen ist im Studienjahr 2005/06 ihre Zahl bereits auf 315 gestiegen. Dort studieren 620,8 Tsd. junge Menschen, was einen Anteil von 31,8\% aller Studierenden ausmacht. Die nichtstaatlichen Hochschulen entstanden zumeist in der Provinz oder in kleineren Städten und bedeuten für die jeweilige Region einen Prestigegewinn. Das Studienangebot umfasst solche Studiengänge wie Finanzen und Ökonomie, Politologie, Ökologie oder Bankwesen, die gerade unmittelbar nach dem Umbruch an den staatlichen Hochschulen fast gar nicht vertreten waren. Inzwischen haben auch hier die staatlichen Hochschulen aufgeholt. Die nichtstaatlichen Hochschulinstitutionen sind relativ klein, haben mit Personalmangel zu kämpfen und bieten ihre Ausbildung in der Regel auf der Ebene des Lizentiats (erster akademische Grad) an. Hinzu kommt die unterschiedliche Qualität des Studienangebots, die von den Fachkreisen und der Öffentlichkeit von elitär bis fragwürdig eingeschätzt wird. Das Studium dort ist gebührenpflichtig. Um eine vergleichbare Bildungsqualität des sich stark diversifizierenden Hochschulwesens in Polen zu gewährleisten, wurde 2002 


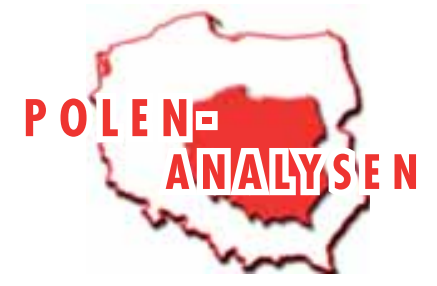

eigens eine Staatliche Akkreditierungskommission (Państwowa Komisja Akredytacyjna) ins Leben gerufen.

\section{Bildungsexpansion}

Infolge der vielfältigen Erneuerungsmaßnahmen und einer Bildungspolitik, die ein hohes Bildungsniveau und die Ausschöpfung des Qualifikationspotentials der Bevölkerung als wesentlich für den Gang des gesellschaftlichen Transformationsprozesses und für den ökonomischen Fortschritt ansieht, setzte in den 1990er Jahren auf der oberen Sekundarstufe eine gezielte Bildungsexpansion und im tertiären Sektor des Bildungssystems durch eine breitere Öffnung des Hochschulzugangs eine Bildungsexpansion "von unten" ein. Sie wurde von folgenden bildungspolitischen Zielen begleitet:

- Reduzierung der Ausbildungskapazitäten in der grundlegenden beruflichen Bildung (auf max. $20 \%$ ) zugunsten weiterführender allgemeiner Schulbildung;

- horizontale Durchlässigkeit auf der oberen Sekundarstufe (zumindest in den ersten beiden Halbjahren der oberen Sekundarschulbildung);

- Durchsetzung der Sekundarschulbildung auf dem Abiturniveau (80\% eines Altersjahrgangs zum Abitur nach dem französischen Vorbild);

- Etablierung eines externen und internen Bewertungs- und Prüfungssystems (Bildungsstandards, Zentralabitur);

- Erhöhung der Quote der Studienanfänger, Studenten und Hochschulabsolventen (auf den OECD Durchschnitt von $50 \%$ - Deutschland $35 \%$ );

- verstärkter Erwerb westlicher Fremdsprachen sowie

- Umsetzung der europäischen Dimension im Bildungswesen.

So konnten im Hochschulwesen die ehemals sehr niedrigen Studentenzahlen vervierfacht werden, von 0,4 Mio. im Studienjahr 1990/91 auf knapp 2 Mio. im Studienjahr 2006/07 und der Studienanfänger von 12,9 \% eines entsprechenden Jahrgangs der 19-24-Jährigen im Studienjahr 1990/91 auf 48,9 \% (Studienjahr 2005/06). Die Erhöhung der in internationalem Vergleich relativ niedrigen Quote hing auch mit der Expansion der Hochschulen zusammen, deren Zahl zu Beginn der Transformation von 112 auf inzwischen 448 (2006/07) gestiegen ist. Diese Steigerung ging vor allem auf die Gründungswelle des nichtstaatlichen Hochschulsektors und der Fachhochschulen (Wyższe Szkoły Zawodowe, »Höhere Berufsschulen«, 2006: 193) zurück. Insgesamt gilt, dass in Polen seit Beginn der Transformation der Übergang von einer »Elite-« zur Massenuniversität vollzogen wurde.

\section{Europäisierung der Hochschulen im Zeichen von Bologna}

Ähnlich wie im übrigen Europa kommen seit dem Ende der 1990er Jahre weltweite Tendenzen, die durch internationale Organisationen wie die Weltbank, die OECD, aber auch die UNESCO induziert werden, auch im polnischen Bildungswesen zum Tragen. Es sind vor allem die neuen Steuerungsmodelle (New Governance), die Qualitätsdebatte, die Herausforderungen der neuen Informations- und Kommunikationstechnologien, das lebenslange Lernen und die Internationalisierung der Hochschulen. Sie überlagern sich mit den Europäisierungsbestrebungen wie Realisierung der europäischen Dimension im Bildungswesen oder Bologna-Prozess, die freilich ebenso als eine mögliche Antwort auf die Herausforderungen der Globalisierung gewertet werden können.

Im Bildungsbereich zeichnete sich nicht erst die 1999 begonnene Reform durch eine gewisse Europakompatibilität aus, wie die rege Teilnahme an den EU-Programmen, verstärkte Kooperation auf der Ebene von Schulund Hochschulpartnerschaften und die bilateralen Aktivitäten in den Euro-Regionen bzw. Grenzregionen zeigten. Ein entsprechend prominentes Beispiel stellt die Gründung der Europauniversität Viadrina in Frankfurt (Oder) zu Beginn der 1990er Jahre dar. Deutlich zum Tragen kommt die europäische Orientierung auch durch Implementierung einiger Elemente sog. Europäischer Bildung im Schulcurriculum, Gründung von Europa-Klubs, Installierung schulinterner und schulübergreifender Projekte, Schaffung spezieller EuropaStudiengänge an den Universitäten, durch einen regen Schüler- und Jugendaustausch oder in der Lehrerfortbildung vor allem in westlichen Fremdsprachen.

Hinsichtlich des Hochschulsektors sind die Fragen der Europäisierung spätestens mit dem Bologna-Prozess virulent geworden, sodass seit 1999 die Entwicklung des polnischen Hochschulsystems dadurch zunehmend beeinflusst wird. Der Bologna-Prozess zielt auf die Architektur eines gemeinsamen europäischen Hochschulraums bis zum Jahre 2010. Im Bereich der Studiengangstrukturen wird durch den Bologna-Prozess erstmals eine konsekutive Zweistufigkeit (zwei aufeinander aufbauende Studienzyklen) und im Idealfall auch die Dreistufigkeit (einschließlich des Promotionsstudiums als eine weitere, nämlich dritte Studienstufe) nicht mehr bloß in national-lokaler Initiative, sondern bewusst gesamteuropäisch angestrebt. Mit der Einführung von Bachelor und Master als reguläre Studienabschlüsse sowie des Systems von Leistungspunkten (Credits) soll die Verständlichkeit und Vergleichbarkeit der akademischen Grade und insgesamt eine Steigerung der Mobilität der Studierenden innerhalb Europas und auch weltweit erreicht werden. 


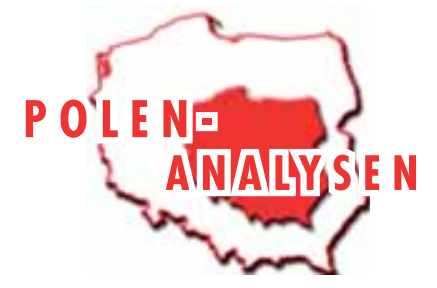

Dieser Teil der akademischen Reformen, nämlich die innere Diversifizierung der Studiengangstrukturen, ist in Polen schon sehr früh und z. T. recht umfassend (etwa $50 \%$ aller Studierenden an nichtstaatlichen und etwa ein Drittel an den staatlichen Hochschulen), also noch vor dem formalen Beginn des Bologna-Prozesses, durchgeführt worden. Für Polen bedeutete die Zweistufigkeit vorrangig eine Transformation der ehemals einheitlichen fünfjährigen Magisterstudiengänge in eine zweistufige Struktur. In Polen dominierte bis zum Eintritt in den Bologna-Prozess weitgehend das sog. Y-Modell, bei dem nach der ersten Phase (in der Regel sechs bis sieben Semester im Lizentiat und sieben bis acht Semester in Ingenieurfächern) entweder die Berufsstudien (ein bis drei Semester, max. bis zu dreieinhalb Jahren) oder das Magisterstudium (vier Semester) folgte. Dabei wird der Übergang von der ersten in die zweite Phase nicht nur völlig unterschiedlich in den einzelnen Hochschulen, sondern auch innerhalb einer Universität oder der Fakultät geregelt. Hinzu kommt, dass die zweite Studienphase für das Gros der Studentenschaft nicht zu einem Hochschul- bzw. Studienortwechsel oder in die Berufstätigkeit führt, wie einige neuere polnische Studien zeigen konnten, was dem eigentlichen Ziel des Bologna-Prozesses entgegen steht, das mit der Stufigkeit auf eine stärkere Mobilität der Studierenden und einen schnelleren Übergang in den Arbeitsmarkt setzt. Demnach besteht trotz der Zweistufigkeit des Studiensystems auch in Polen immer noch ein gewisser Anpassungsbedarf an die Bologna-Ziele, zumal, wie polnische Experten bemerken, vielmehr die Bezeichnungen und weniger die tatsächlichen Studiengänge geändert wurden.

So gibt der Länderbericht 2004, der regelmäßig im Kontext der Treffen europäischer Bildungsminister vorgelegt wird, an, dass erst knapp die Hälfte aller staatlichen Hochschulen, ein Drittel nichtstaatlicher Hoch- schulen und knapp 10 \% der Fachhochschulen mindestens die Hälfte aller Studiengänge auf die neue B.A./ M.A.-Struktur umgestellt haben. Vor allem die Einführung des Kreditpunktesystems (ECTS = European Credit Transfer System), das die Studienleistungen zertifiziert, und der Diploma Supplements scheint noch in den »Kinderschuhen« zu stecken. Zwar trifft der BolognaProzess beim polnischen Hochschulministerium und bei der Rektorenkonferenz polnischer Hochschulen (Konferencja Rektorów Akademickich Szkół Polskich) auf grundsätzliche Zustimmung, doch gibt es auch hier skeptische Stimmen, die den Verlust eigener spezifischer Charakteristika, wie z. B. des bis dahin in Polen zumeist breit angelegten Grundstudiums erwarten. Die akademische Welt, vornehmlich der renommierten Hochschuleinrichtungen, befürchtet eine Nivellierung der Leistungen nach unten und sieht teilweise die Qualität des Studiums durch die verkürzten Studienzeiten gefährdet. Zudem herrscht im polnischen Hochschulwesen überwiegend eine konventionelle Vorstellung, die ein vollwertiges Hochschulstudium immer noch mit einem fünführigen Magisterstudium konnotiert. Freilich sieht man im Bologna-Projekt bei aller Bereitschaft, sich den Internationalisierungsprozessen zu stellen, ähnlich wie in Deutschland, weniger die Europäisierungsbemühungen als die bloße Übertragung angelsächsischer Muster.

Dem internationalen Wettbewerbsdruck auf dem Hochschul- und Wissenschaftssektor muss sich die polnische Bildungspolitik stellen und zwar nicht nur mit Blick auf Bologna, also unter dem Gesichtspunkt der Attraktivität polnischer Hochschulen für ausländische Studierende, sondern auch mit Blick auf den nicht zu vernachlässigenden Brain-Drain vor allem gut ausgebildeter junger Akademiker.

\section{Über die Autorin}

PD Dr. Sonja Steier, Vergleichende Erziehungswissenschaft, Institut für Pädagogik, Ruhr-Universität Bochum. Leiterin der Fachgruppe Pädagogik in der Deutschen Gesellschaft für Osteuropakunde. Forschungsschwerpunkte: bildungspolitische und pädagogische Entwicklungen in Mittel- und Osteuropa im 20.Jh. bis zur Gegenwart mit dem Länderschwerpunkt Polen und Russland, polnische Wissenschafts- und Bildungsgeschichte, Ost-West-Vergleich und Internationalisierungsprozesse in Bildungssystemen.

\section{Literaturauswahl}

Internetquellen (Stand: 31. März 2008)

- http:// w w w.men.gov.pl (Portal des polnischen Bildungsministeriums)

- $\quad$ http:// w w w .na uka.gov.pl (Portal des polnischen Ministeriums für Wissenschaft und Hochschulwesen)

- http:// w w w.stat.gov.pl (Portal des polnischen Statistischen Hauptamts)

- http:// w w w.ow w z.de/ science_news.html? \&L=L (Ost-West-Wissenschaftszentrum, Kassel) 


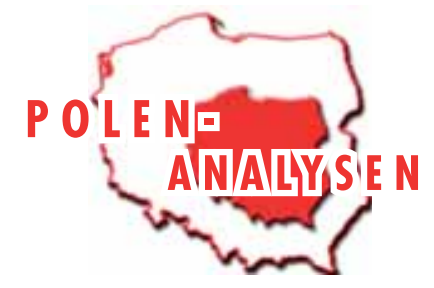

\section{Die Abschaffung der Habilitation in Polen in der Diskussion}

Fryderyk Zoll, Krakau

\section{Polen und Deutschland - oberflächliche Ähnlichkeit}

Bei einer oberflächlichen Betrachtung sieht der akademische Werdegang in Polen ähnlich wie in Deutschland aus. Die Erlangung der Doktorwürde und später die Habilitation sind Meilensteine einer wissenschaftlichen Karriere. Diese Ähnlichkeit ist aber oberflächlich und deckt eine wesentlich andere Wirklichkeit der polnischen akademischen Landschaft zu. Das heißt nicht, dass die polnischen Doktortitel oder die polnische Habilitation nicht vergleichbar wären, wenn es um die wissenschaftliche Qualität geht. Man bemängelt zwar manchmal die sinkende Qualität polnischer Promotionen, aber eigentlich ist hier keine große Krise festzustellen. Zwei Gutachten bei den Promotionen (eines immer aus einer anderen Universität) und die Stellungnahme des Doktorvaters (anders als in Deutschland wird das Erstgutachten nicht von ihm erstellt) sorgen dafür, dass ein gewisser Grad an Qualität für die Erlangung der Doktorwürde gesichert wird. Bei der Habilitation sind sogar vier Gutachten erforderlich: Zwei Gutachter werden von der Fakultät ernannt (einer davon muss aber ein externer Gutachter sein), weitere zwei von der Zentralen Qualifikationsbehörde (Centralna Komisja Kwalifikacyjna) - ein staatliches Organ, das auch von Wissenschaftlern besetzt wird.

Der Kernunterschied liegt in dem Weg, der nach der Habilitation beschritten wird. In Polen ist kein eigentliches Berufungssystem bekannt. Ein habilitierter Wissenschaftler, der bereits an der Universität angestellt war (als Assistent und dann später als Adjunkt), wird sein Dienstverhältnis fortsetzen und zwar an der selben Universität. Am Anfang trägt er zwar den etwas abwertenden Titel des "habilitierten Adjunkten«. Darunter verstecken sich aber fast alle Rechte, die jedem sog. selbständigen Wissenschaftler zustehen - er wird ein volles Mitglied des Fakultätsrates, er hält eigene Vorlesung und betreut Doktoranden. An einigen Universitäten ist es ihm erlaubt, einen Lehrstuhl zu leiten, an anderen nicht - was aber nicht so ausschlaggebend ist. Lehrstühle sind in Polen eher kleine Institute mit vielen Professoren (oder "selbständigen Wissenschaftlern«), und ein Lehrstuhlinhaber zu sein, ist oft eine lästige Verwaltungsaufgabe. Mit den Aufgaben eines deutschen Lehrstuhls hat dies wenig zu tun.

Nach einer bestimmten "Karenz« wird der habilitierte Adjunkt Universitätsprofessor. Der Gesetzgeber hat sehr viel Mühe darauf verwendet, unterschiedliche Arten von Professorenstellen zu entwickeln. Die erste Stufe heißt Universitätsprofessor. Es ist eine Stelle an der Universität. Dann können die Wissenschaftler den Titel des Professors vom Staatspräsidenten verliehen bekommen. Darüber hinaus gibt es außerordentliche und ordentliche Professoren. Diese Unterscheidung ist zwar österreichischen Ursprungs, hat aber ansonsten nichts mehr damit zu tun. Es ist eher eine Frage der Ehre und vielleicht einer etwas differenzierteren Entlohnung. Eine gewisse Zahl von Professoren und Wissenschaftlern nach der Habilitation zu haben, ist für die Hochschulen lebenswichtig, denn davon hängt die Akkreditierung ab sowie das Promotions- und Habilitationsrecht. Anders als in Deutschland bedeutet eine Professorenstelle noch nicht, eine sichere Stellung auf Lebenszeit erlangt zu haben. An einer staatlichen Universität sind die Gehälter wesentlich niedriger, als dies berechtigterweise zu erwarten wäre. Daher ist beispielsweise fast jeder Professor einer juristischen Fakultät entweder noch an einer anderen, oft privaten Hochschule tätig oder betreibt eine volle juristische Tätigkeit in der Praxis.

An manchen "guten « juristischen Fakultäten steigt mit zunehmender Zahl der Habilitationen von eigenen Mitgliedern des akademischen Mittelbaus automatisch die Zahl der selbständigen Wissenschaftler, die auch grundsätzlich nicht entlassen werden können. Sie bleiben an der eigenen Universität, und das Bemühen geht dahin, eine ausreichende Zahl der obligatorischen Unterrichtsstunden für sie zu gewährleisten. An solchen Universitäten wächst die Zahl der Professoren. Es ist nicht Ungewöhnliches, dass für etwa 4.000 Studierende über 60 »selbständige Wissenschaftler« zur Verfügung stehen. Allerdings betrachten sie die Arbeit an der Universität nicht selten als einen Nebenjob und als eine reine Prestigesache, hilfreich für ihre anderen Tätigkeiten. Dieses System stößt allmählich an die Grenzen des Erträglichen. Die Zahl der Professoren in solchen Fakultäten kann nicht unbeschränkt wachsen. Dagegen gibt es an anderen Universitäten und privaten juristischen Fakultäten ein anderes Problem. Sie verfügen über eine kaum ausreichende Zahl an "selbständigen Wissenschaftlern«, die am sogenannten ersten Arbeitsplatz (nur dieser zählt für die Akkreditierung) arbeiten möchten. Die Habilitierten an den Prestigefakultäten möchten diese trotz des niedrigen Gehalts nicht verlassen - sie nutzen eben deren Prestige und verdienen das Geld anderswo.

\section{Was sollte man tun?}

Es stellt sich nun die Frage, was in einer solchen Situation getan werden sollte, um die Lage deutlich zu ver- 


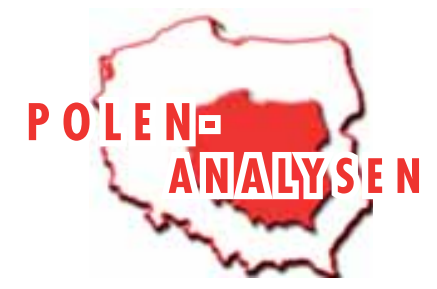

bessern. Die Antwort scheint auf der Hand zu liegen. Die Zahl der Stellen an den Fakultäten müsste festgelegt werden, und sie müssten mit denjenigen besetzt werden, die sich auf die Aktivitäten an der Universität voll konzentrieren möchten. Es sollte darüber hinaus ein Hausberufungsverbot eingeführt werden. Die Professoren, deren Zahl durch die Festlegung einer Obergrenze gekappt werden sollte, sollten entschieden besser entlohnt werden. Das wäre auch im Rahmen des bestehenden Mittelumfangs zu erreichen. Die enorm hohe Zahl von Professoren an den Prestigeuniversitäten könnte ohne Schaden um 2/3 reduziert werden, wenn das restliche Drittel gut bezahlt würde und an der Universität unbeschränkt und viel besser motiviert zum Einsatz käme. Als Folge eines Hausberufungsverbot würden sich die hoch qualifizierten Habilitierten auch an die anderen Universitäten bewerben, so dass sich die intellektuellen Ressourcen besser und gerechter verteilen würden. Das vorgeschlagene »deutsche Modell» ist vielleicht keine ideale Lösung, aber seine Übernahme kann sich für die polnischen Universitäten eignen, weil das Konzept der Universität in Polen dem deutschen Konzept relativ nah steht.

\section{Reformbemühungen}

Die neue polnische Regierung von Ministerpräsident Donald Tusk hat die Notwendigkeit einer Reform im Hochschulbereich erkannt. Die Ministerin für Wissenschaft und Hochschulwesen, Barbara Kudrycka, die vorher Rektorin an einer privaten Hochschule war, hat eine Arbeitsgruppe eingerichtet, die vor kurzem als erste Ergebnisse ihrer Tätigkeit die Grundzüge einer Reform der Öffentlichkeit vorgelegt hat. Den Kern des Programms macht die Abschaffung der Habilitation aus. Die Argumente für diesen Schritt kommen vor allem aus dem Bereich der »harten "Wissenschaftsdisziplinen (Medizin u.a.), z.B. dass die Habilitation die Karriere von jungen Wissenschaftlern übermäßig erschwere, die Alten den Jungen im Wege stünden usw. Diese Beobachtungen sind nicht ganz falsch. In vielen Bereichen, wie z.B. der Medizin, lassen sich derartige Vorfälle wahrnehmen. Professoren bilden dort eine Art Korporation, die sich gegen Einflüsse von außen abschließt. Aus der
Sicht der Juristen sieht die Lage jedoch völlig anders aus. Die Zahl der erfolgreichen Habilitationen steigt, und immer Jüngere erlangen diese Würde. Es gibt keine künstlichen Schwellen. Der Druck, möglichst schnell zu habilitieren, wird erhöht. Die Abschaffung der Habilitation wäre hier kein Heilmittel, wenn nicht die gesamte Struktur grundsätzlich verändert würde. Das Problem liegt darin, dass das heutige Modell keinen Anreiz zur Mobilität der Wissenschaft schafft. Die Professoren versinken in den zahlreichen Nebentätigkeiten, die faktisch zu Haupttätigkeiten werden.

\section{Warum ist eine echte Reform so schwer?}

Die eventuelle Abschaffung der Habilitation würde die Lösung des Problems nur erschweren. Der Ansatz zur Lösung liegt vielmehr in der Etablierung eines fairen Selektionsprozesses bei der Besetzung von Professorenstellen, deren Zahl festgelegt werden muss. Man muss erzwingen, dass der Universitätsberuf wieder zur Vollbeschäftigung wird, und zwar materiell und nicht nur formell. Wenn die Reform darauf beschränkt wird, dass die Doktoren Professoren werden, ändert sich einfach nichts. Es besteht aber kein ernsthafter Wille, die verkrusteten Strukturen wirklich zu verändern. Sehr vielen erfolgreichen Anwälten, Richtern usw., die zugleich Professoren sind, kommt das heutige Modell sehr entgegen. Jede Reform würde bedeuten, dass alle diese gut etablierten Akademiker verifiziert werden müssten oder zumindest eine Entscheidung zu treffen hätten, welche Tätigkeit sie letztendlich fortführen wollen. Das würde in vielen Fällen eine Veränderung der bisherigen Lebensweise erzwingen und eine viel größere Mobilität erfordern. Die Abschaffung der Habilitation würde den privaten Hochschulen das Leben erleichtern, weil sie deren Kosten senken würde. Es wäre aber eine Scheinreform, die den Unwillen verdecken würde, die wirklich entscheidenden Schritte zu einer Reform zu unternehmen. Das etablierte akademische Milieu ist mit dem heutigen System zufrieden, weil es ihm einen ruhigen Schlaf ermöglicht. Und nach einer Abschaffung der Habilitation würde dieser Schlaf schon einige Jahre früher beginnen können.

\section{Über den Autor}

Fryderyk Zoll (geb. 1970) ist Professor für Privatrecht an der Krakauer Jagiellonen-Universität. Er zählt zu den profiliertesten jüngeren polnischen Rechtswissenschaftlern. In deutscher Sprache veröffentlichte er u.a. als Herausgeber: Einführung in das polnische Recht (München 2005, zus. mit Marc Liebscher). 


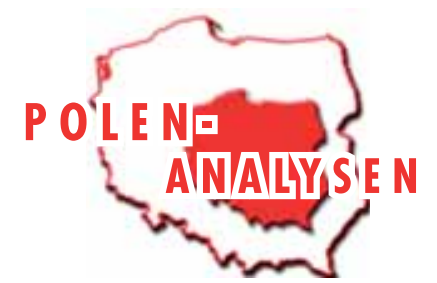

Tabellen und Grafiken

Das polnische Hochschulsystem

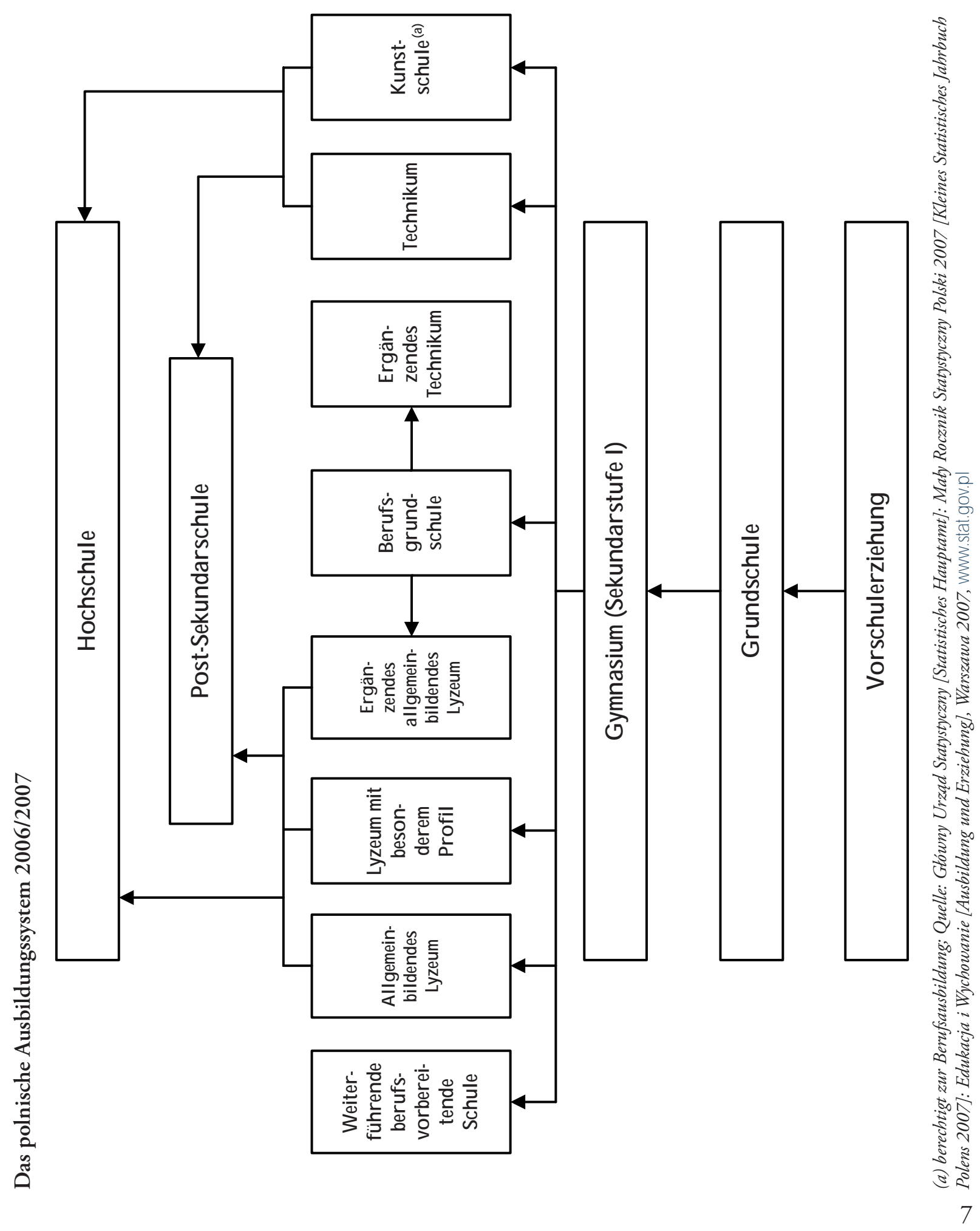




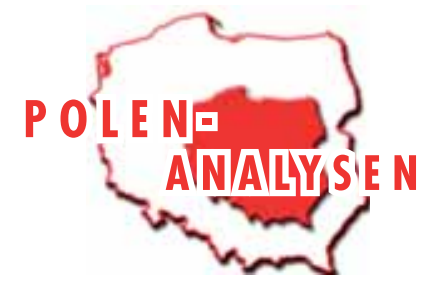

Hochschulen nach Typen

\begin{tabular}{|c|c|c|c|c|c|}
\hline & $\begin{array}{c}\text { 2006/07 } \\
\text { Hochschulen }\end{array}$ & $\begin{array}{c}2005 / 06 \\
\text { Studierende in } \\
\text { Tsd. }\end{array}$ & $\begin{array}{c}2006 / 07 \\
\text { Studierende in } \\
\text { Tsd. }\end{array}$ & $\begin{array}{c}2004 / 05 \\
\text { Absolventen in } \\
\text { Tsd. }\end{array}$ & $\begin{array}{c}2005 / 06 \\
\text { Absolventen in } \\
\text { Tsd. }\end{array}$ \\
\hline insgesamt & 448 & 1953,8 & 1941,4 & 391,5 & 394,0 \\
\hline reguläres Tagesstudium & - & 950,0 & 950,4 & 156,8 & 167,4 \\
\hline $\begin{array}{l}\text { Abend-, Wochenend-, } \\
\text { Fernstudium }\end{array}$ & - & 1003,8 & 991,1 & 234,7 & 226,6 \\
\hline Universitäten & 18 & 563,1 & 550,5 & 116,8 & 115,9 \\
\hline Technische Hochschulen & 22 & 331,0 & 318,9 & 56,3 & 55,7 \\
\hline Landwirtschaftl. Hochschulen & 8 & 107,7 & 92,0 & 18,4 & 16,6 \\
\hline Wirtschaftshochschulen & 95 & 407,7 & 406,2 & 94,9 & 88,0 \\
\hline Pädagog. Hochschulen & 17 & 111,8 & 117,4 & 30,2 & 32,7 \\
\hline Medizinische Akademien & 9 & 48,8 & 53,1 & 7,8 & 9,9 \\
\hline Hochschulen für Seefahrt & 2 & 11,5 & 10,5 & 2,4 & 2,4 \\
\hline Sportakademien & 6 & 28,2 & 29,0 & 5,2 & 5,7 \\
\hline Kunsthochschulen & 21 & 15,4 & 14,9 & 2,7 & 2,8 \\
\hline Theologische Hochschulen & 14 & 10,4 & 10,7 & 2,1 & 2,7 \\
\hline $\begin{array}{l}\text { Schulen des Ministeriums für } \\
\text { Verteidigung }\end{array}$ & 5 & 12,0 & 11,7 & 2,4 & 1,8 \\
\hline $\begin{array}{l}\text { Schulen des Ministeriums für } \\
\text { Inneres u. Verwaltung }\end{array}$ & 2 & 2,0 & 2,1 & 0,5 & 0,5 \\
\hline andere Hochschulen & 229 & 304,2 & 324,5 & 51,8 & 59,4 \\
\hline Berufshochschulen* & 193 & 224,7 & 244,5 & 34,5 & 42,0 \\
\hline $\begin{array}{l}\text { andere nichtstaatliche Hoch- } \\
\text { schulen }\end{array}$ & 36 & 79,5 & 80,0 & 17,3 & 17,4 \\
\hline
\end{tabular}

* im Text von Sonja Steier in dieser Ausgabe auch als »Fachhochschulen« bezeichnet.

Quelle: Główny Urzad Statystyczny [Statistisches Hauptamt]: Maty Rocznik Statystyczny Polski 2007 [Kleines Statistisches Jahrbuch Polens 2007]: Edukacja $i$ Wychowanie [Ausbildung und Erziehung], Warszawa 2007, w w w.stat.go v.pl

Nichtstaatliche Hochschulen

\begin{tabular}{|c|c|c|c|c|c|c|c|c|}
\hline & \multicolumn{2}{|c|}{$1995 / 96$} & \multicolumn{2}{|c|}{$2000 / 01$} & \multicolumn{4}{|c|}{$2005 / 06$} \\
\hline & Schulen & $\begin{array}{l}\text { Studie- } \\
\text { rende }\end{array}$ & Schulen & $\begin{array}{l}\text { Studie- } \\
\text { rende }\end{array}$ & Schulen & $\begin{array}{l}\text { Studie- } \\
\text { rende }\end{array}$ & $\begin{array}{c}\text { Absol- } \\
\text { venten }^{(a)}\end{array}$ & Lehrkräfte \\
\hline insgesamt & 80 & 89.399 & 195 & 472.340 & 315 & 620.800 & 129.227 & 16.862 \\
\hline Universitäten & 1 & 13.261 & 1 & 16.891 & 1 & 20.654 & 4.160 & 1.120 \\
\hline $\begin{array}{l}\text { Technische } \\
\text { Hochschulen }\end{array}$ & 5 & 2.678 & 5 & 9.624 & 4 & 9.343 & 1.992 & 314 \\
\hline $\begin{array}{l}\text { Landwirtschaftliche } \\
\text { Hochschulen }\end{array}$ & - & - & 1 & 1.600 & 1 & 1.675 & 317 & 36 \\
\hline Wirtschaftshochschulen & 46 & 43.547 & 89 & 296.299 & 90 & 331.104 & 76.769 & 7.451 \\
\hline Pädagog. Hochschulen & 5 & 14.098 & 11 & 40.519 & 11 & 43.189 & 12.038 & 807 \\
\hline Kunsthochschulen & 3 & 388 & 4 & 920 & 4 & 1.152 & 265 & 87 \\
\hline $\begin{array}{l}\text { Theologische } \\
\text { Hochschulen }\end{array}$ & 9 & 5.646 & 14 & 8.402 & 12 & 9.430 & 1.979 & 821 \\
\hline Berufshochschulen* & - & - & 45 & 31.854 & 161 & 124.709 & 14.366 & 4.101 \\
\hline $\begin{array}{l}\text { andere Hochschulen } \\
\text { (mit Universitätsprofil) }\end{array}$ & 11 & 9.781 & 25 & 66.231 & 31 & 79.544 & 17.341 & 2.125 \\
\hline
\end{tabular}

(a) Für 2004/05; * im Text von Sonja Steier in dieser Ausgabe auch als »Fachhochschulen« bezeichnet.

Quelle: Gtówny Urząd Statystyczny [Statistisches Hauptamt]: Rocznik Statystyczny 2006 [Statistisches Jahrbuch 2006] Edukacja $i$ Wychowanie [Ausbildung und Erziehung], Warszawa 2006. 


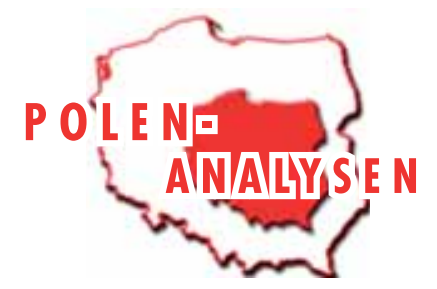

Ausländische Studierende und Absolventen

\begin{tabular}{|l|c|c|c|c|}
\hline & $1995 / 96$ & $2000 / 01$ & $2005 / 06$ & $2006 / 07$ \\
\hline Studierende & 5.202 & 6.563 & 10.092 & 11.752 \\
\hline $\begin{array}{l}\text { davon polnischer } \\
\text { Herkunft }\end{array}$ & 2.454 & 3.618 & 3.723 & 3.510 \\
\hline $\begin{array}{c}\text { Absolventen } \\
\text { davon polnischer } \\
\text { Herkunft }\end{array}$ & $697^{(\text {a) }}$ & 892 & 1.306 & o.A. \\
\hline
\end{tabular}

(a) Angabe für 1995

Quelle: Główny Urzad Statystyczny [Statistisches Hauptamt]: Maty Rocznik Statystyczny Polski 2007 [Kleines Statistisches Jahrbuch Polens 2007]: Edukacja i Wychowanie [Ausbildung und Erziehung], Warszawa 2007, w w w.stat.gov.pl und Główny Urząd Statystyczny [Statistisches Hauptamt]: Rocznik Statystyczny 2006 [Statistisches Jahrbuch 2006] Edukacja i Wychowanie [Ausbildung und Erziehung], Warszawa 2006.

Studierende und Absolventen an den Hochschulen

\begin{tabular}{|c|c|c|c|c|c|}
\hline Studienrichtungen ${ }^{(a)}$ & $\begin{array}{c}2005 / 06 \text { Stu- } \\
\text { dierende in } \\
\text { Tsd. }\end{array}$ & $\begin{array}{l}\text { 2006/07 Stu- } \\
\text { dierende in } \\
\text { Tsd. }\end{array}$ & $\begin{array}{c}2006 / 07 \\
\text { Studentinnen } \\
\text { in } \%\end{array}$ & $\begin{array}{l}2004 / 05 \mathrm{Ab}- \\
\text { solventen in } \\
\text { Tsd. }\end{array}$ & $\begin{array}{l}2005 / 06 \mathrm{Ab}- \\
\text { solventen in } \\
\text { Tsd. }\end{array}$ \\
\hline insgesamt & 1953,8 & 1941,4 & 56,4 & 391,5 & 394,0 \\
\hline Pädagogische & 250,6 & 236,7 & 70,7 & 60,9 & 59,9 \\
\hline Künstlerische & 22,2 & 23,2 & 64,3 & 3,2 & 3,7 \\
\hline Humanistische $^{(b)}$ & 156,5 & 175,1 & 71,9 & 27,8 & 33,1 \\
\hline Sozialwissenschaftliche & 264,7 & 272,5 & 64,3 & 56,7 & 56,7 \\
\hline Journalistische u. medienwiss. & 16,8 & 18,4 & 67,4 & 3,5 & 3,7 \\
\hline $\begin{array}{l}\text { Wirtschafts- u. } \\
\text { verwaltungswissenschaftl. }\end{array}$ & 501,8 & 470,3 & 59,7 & 122,4 & 111,7 \\
\hline Juristische & 54,6 & 59,0 & 54,6 & 8,7 & 8,2 \\
\hline Biologische $e^{(c)}$ & 13,7 & 13,5 & 80,8 & 2,7 & 3,1 \\
\hline Physikalische $e^{(\mathrm{d})}$ & 34,5 & 32,2 & 58,0 & 5,9 & 6,1 \\
\hline Mathematik u. Statistik & 16,0 & 16,0 & 61,5 & 2,9 & 3,3 \\
\hline Informatik & 103,9 & 102,2 & 10,9 & 15,5 & 17,2 \\
\hline Technisches Ingenieurwesen & 153,5 & 148,1 & 18,0 & 22,3 & 22,7 \\
\hline Produktion und Verarbeitung & 28,1 & 28,1 & 48,3 & 3,8 & 3,9 \\
\hline Architektur und Bauwesen & 56,5 & 57,7 & 36,4 & 7,6 & 8,1 \\
\hline $\begin{array}{l}\text { Land- u. Forstwirtschaft, } \\
\text { Fischereiwesen }\end{array}$ & 39,7 & 39,3 & 51,2 & 6,5 & 6,3 \\
\hline Tiermedizin & 4,3 & 4,4 & 65,1 & 0,6 & 0,6 \\
\hline Medizin & 92,1 & 103,4 & 76,1 & 14,0 & 19,0 \\
\hline Sozialarbeit & 1,9 & 0,1 & 89,7 & 0,5 & - \\
\hline Personalwesen & 64,2 & 67,5 & 62,7 & 11,6 & 13,0 \\
\hline Transportwesen & 16,0 & 16,0 & 13,2 & 2,0 & 2,3 \\
\hline Umweltschutz & 55,7 & 54,5 & 51,5 & 10,5 & 10,6 \\
\hline Sicherheit u. Gefahrenabwehr & 6,5 & 3,4 & 16,0 & 1,9 & 0,9 \\
\hline
\end{tabular}

(a) nach der International Standard Classification of Education (ISCED '97)

(b) inklusiv Theologie

(c) u.a. Biologie, Botanik, Biochemie, Toxikologie, Genetik, Zoologie

(d) u.a. Astronomie, Physik, Chemie, Geologie

Quelle: Główny Urząd Statystyczny [Statistisches Hauptamt]: Mały Rocznik Statystyczny Polski 2007 [Kleines Statistisches Jahrbuch

Polens 2007]: Edukacja i Wychowanie [Ausbildung und Erziehung], Warszawa 2007, w w w.stat.go v.pl 


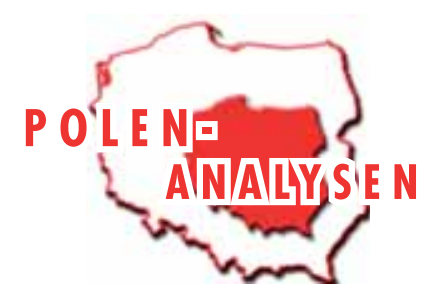

Anzahl der Stipendienempfänger im Hochschuljahr 2006/07

\begin{tabular}{|l|c|c|}
\hline & in Tausend & in \% \\
\hline insgesamt $^{(a)}$ & 562,7 & 29,0 \\
\hline Sozialstipendien & 336,6 & 17,3 \\
$\begin{array}{l}\text { Stip. für besondere wissenschaftl. oder } \\
\text { sportl. Leistungen }\end{array}$ & 263,5 & 13,6 \\
\hline
\end{tabular}

(a) Bei der Einteilung nach Stipendiumsarten waren Mehrfachnennungen möglich.

Quelle: Główny Urzad Statystyczny [Statistisches Hauptamt]: Maty Rocznik Statystyczny Polski 2007 [Kleines Statistisches Jahrbuch

Polens 2007 ]: Edukacja $i$ Wychowanie [Ausbildung und Erziehung], Warszawa 2007, w w w.stat.go v.p I

\section{Chronik}

\section{Vom 18. März bis zum 31. März 2008}

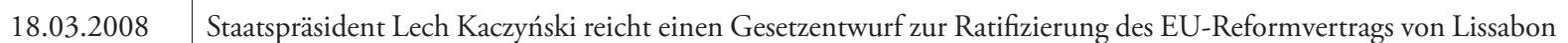
im Sejm ein, der die Bedingungen der Oppositionspartei Recht und Gerechtigkeit (Prawo i Sprawiedliwość - PiS) zur Ratifizierung beinhaltet. Festgeschrieben wird darin die Notwendigkeit eines Konsens zwischen dem Staatspräsidenten, der Regierung und dem Parlament im Falle eines Verzichts auf den Ioannina-Kompromiss, auf das britische Protokoll zur Grundrechtecharta (das diese einschränkt) und auf die Festlegung, dass die polnische Gesetzgebung u.a. in Fragen der öffentlichen Moral souverän ist.

19.03.2008 Die Bundesregierung beschließt die Einrichtung eines »Sichtbaren Zeichens« als Dokumentationszentrum zu Flucht und Vertreibungen. Es soll dem Deutschen Museum in Berlin als unselbständige Stiftung angegliedert werden und in einer Dauerausstellung über die Vertreibung der Deutschen nach dem 2. Weltkrieg informieren. Über die von Deutschen verursachten Vertreibungen soll ebenfalls informiert werden. Dabei wird eine Zusammenarbeit mit den Nachbarländern angestrebt. Die polnische Regierung wird sich an diesem Projekt nicht beteiligen; sollten polnische Experten daran mitarbeiten, so tun sie dies nicht im Namen der Regierung.

20.03.2008 Staatspräsident Lech Kaczyński spricht sich deutlich für die Ratifizierung des EU-Reformvertrags von Lissabon auf parlamentarischem Weg aus, allerdings soll der polnische Verhandlungserfolg von Lissabon, der sich im Gesetzentwurf von Recht und Gerechtigkeit (Prawo i Sprawiedliwość - PiS) widerspiegelt, im Ratifizierungsgesetz betont werden. Er könne sich nicht vorstellen, dass Polen der Annahme des Vertrags durch die Europäische Union im Wege stehe; Polen sei dafür ein viel zu europafreundliches Land. Darüber hinaus widerspricht er Einschätzungen der letzten Tage, er werde die Ratifizierung im Falle einer Verabschiedung durch den Sejm ablehnen. Hintergrund dieser Vermutung ist u.a. eine Fernsehansprache des Staatspräsidenten vom 17.03., in der er einen eigenen Entwurf des Ratifizierungsgesetzes ankündigt. Illustriert wurde der Fernsehauftritt u.a. mit Bildern des EU-Gipfels in Lissabon, einer Hochzeit von Homosexuellen und einer Landkarte von Deutschland in den Grenzen von 1937. Außerdem wurden Bundeskanzlerin Angela Merkel und die CDU-Abgeordnete und Präsidentin des Bundes der Vertriebenen (BdV), Erika Steinbach, eingespielt.

20.03.2008 Während seines Besuchs in Slowenien teilt Ministerpräsident Donald Tusk mit, Polen werde sich der Wiederaufnahme von Gesprächen über ein Partnerschafts- und Kooperationsabkommen zwischen der Europäischen Union und Russland nicht mehr widersetzen, da erstens die polnischen Forderungen zur Aufhebung des russischen Embargos für polnische Fleischprodukte erfüllt worden seien und zweitens die Wiederaufnahme des Exports polnischer pflanzlicher Produkte nach Russland vereinbart worden sei.

25.03.2008 Auf einer Pressekonferenz während seines Besuchs in Ungarn betont Staatspräsident Lech Kaczyński, es gebe in Polen keinen grundsätzlichen Streit über die Ratifizierung des EU-Reformvertrags von Lissabon, sondern nur über den Inhalt des Ratifizierungsgesetzes.

26.03.2008 Der ehemalige stellvertretende Außenminister und Abgeordnete von Recht und Gerechtigkeit (Prawo i Sprawiedliwość - PiS), Karol Karski, äußert in einem Radio-Interview die Vermutung, Ministerpräsident Donald Tusk habe Bundeskanzlerin Angela Merkel versprochen, auf den Ioannina-Mechanismus zu verzichten, der in eine Zusatzerklärung zum EU-Reformvertrag von Lissabon aufgenommen wurde. Es sei daher logisch, dass sich die Regierungspartei Bürgerplattform (Platforma Obywatelska - PO) dagegen wehre, die Regierung bindende Bedingungen für den Verzicht auf den Ioannina-Mechanismus in das Ratifizierungsgesetz für den Reformvertrag aufzunehmen. 


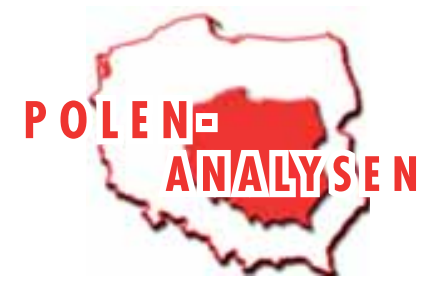

\begin{tabular}{|c|c|}
\hline 26.03.2008 & $\begin{array}{l}\text { Der Abgeordnete von Recht und Gerechtigkeit (Prawo i Sprawiedliwość - PiS) Jacek Kurski teilt in einem Interview } \\
\text { mit, er übernehme die Verantwortung für die Bilder von Bundeskanzlerin Angela Merkel und der CDU-Abge- } \\
\text { ordneten und Präsidentin des Bundes der Vertriebenen (BdV), Erika Steinbach, mit denen der Fernsehauftritt von } \\
\text { Staatspräsident Lech Kaczyński am 17.03. zur Ratifizierung des EU-Reformvertrags von Lissabon illustriert wurde. } \\
\text { Lech Kaczyński habe lediglich gewusst, dass seine Rede multimedial untermalt werde, er habe aber die konkrete } \\
\text { Umsetzung nicht gekannt. Einige Szenen, darunter die oben genannte, hätten ihm nicht gefallen. }\end{array}$ \\
\hline 27.03.2008 & $\begin{array}{l}\text { Nach einem Gespräch über das Ratifizierungsgesetz zum EU-Reformvertrag von Lissabon, in dessen Verlauf } \\
\text { Ministerpräsident Donald Tusk Staatspräsident Lech Kaczyński Rechtsgutachten vorgestellt hatte, die darlegen, dass } \\
\text { der vom Staatspräsidenten eingebrachte Gesetzentwurf nicht verfassungsgemäß ist, zeigen sich beide zuversichtlich } \\
\text { hinsichtlich einer schnellen Einigung über die Vertragsratifizierung im Sejm und im Senat. }\end{array}$ \\
\hline 27.03.2008 & $\begin{array}{l}\text { Ministerpräsident Donald Tusk teilt mit, dass er nicht an den Eröffnungsfeierlichkeiten der Olympischen Sommer- } \\
\text { spiele in Peking im August teilnehmen werde. }\end{array}$ \\
\hline 28.03.3008 & $\begin{array}{l}\text { Nach einer Sitzung der Fraktion von Recht und Gerechtigkeit (Prawo i Sprawiedliwość- PiS) gibt deren Vorsitzender, } \\
\text { Przemysław Gosiewski, bekannt, dass die Fraktion ihre Zustimmung zum Ratifizierungsgesetz für den EU-Reform- } \\
\text { vertrag von Lissabon davon abhängig machen werde, ob die von PiS vorgeschlagenen sog. Absicherungsmechanismen } \\
\text { u.a. für den Ioannina-Kompromiss und die eingeschränkte Grundrechtecharta berücksichtigt werden. }\end{array}$ \\
\hline 29.03 & $\begin{array}{l}\text { Der Vorsitzende der Demokratischen Linksallianz (Sojusz Lewicy Demokratycznej-SLD), Wojciech Olejniczak, } \\
\text { teilt die Aufkündigung der Zusammenarbeit mit der Demokratischen Partei (Partia Demokratyczna - PD) im } \\
\text { Rahmen des Parteienbündnisses Linke und Demokraten (Lewica i Demokraci - LiD) mit. LiD ist mit } 53 \text { Abge- } \\
\text { ordneten im Sejm vertreten. Einer der Gründe sei die liberale Ausrichtung des Wirtschaftsprogramms der PD, mit } \\
\text { der die SLD nicht übereinstimme. }\end{array}$ \\
\hline 30.03 .2008 & $\begin{array}{l}\text { Auf einer Pressekonferenz informiert Ministerpräsident Donald Tusk über die Ergebnisse des Gesprächs mit } \\
\text { Staatspräsident Lech Kaczyński über das Ratifizierungsgesetz für den EU-Reformvertrag von Lissabon am Vortag. } \\
\text { Demnach soll der Sejm über den von der Regierung eingebrachten Gesetzentwurf zur Ratifizierung entscheiden. } \\
\text { Gleichzeitig soll vom Sejmpräsidium eine Entschließung erarbeitet werden, die die Forderungen der Fraktion von } \\
\text { Recht und Gerechtigkeit (Prawo i Sprawiedliwość - PiS) weitgehend übernimmt. Diese haben den Charakter einer } \\
\text { Deklaration, so dass sich hieraus keine Diskussion um ihre Verfassungsmäßigkeit ergeben könne. Darüber hinaus } \\
\text { habe man sich darauf geeinigt, im Sejm eine Neufassung des Gesetzes vom 11.11.2004 über die Beziehungen des } \\
\text { Sejm, des Senats und des Staatspräsidenten zur Europäischen Union zu erarbeiten. }\end{array}$ \\
\hline 31.03 .2008 & $\begin{array}{l}\text { Das Präsidium des Sejm beschließt, am nächsten Tag auf einer Sondersitzung über den Gesetzentwurf zur Ratifi- } \\
\text { zierung des EU-Reformvertrags von Lissabon abzustimmen. }\end{array}$ \\
\hline
\end{tabular}




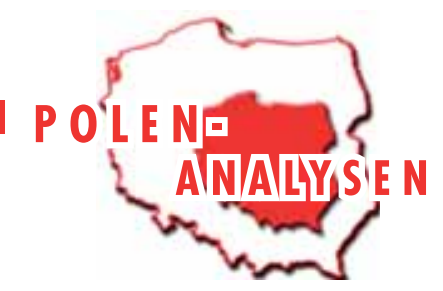

Über die Polen-Analysen

Die Polen-Analysen erscheinen zweimal monatlich als E-Mail-Dienst. Sie werden gemeinsam vom Deutschen PolenInstitut Darmstadt, der Bremer Forschungsstelle Osteuropa und der Deutschen Gesellschaft für Osteuropakunde herausgegeben. Die Herausgeber danken der BSH Bosch und Siemens Hausgeräte GmbH München und dem Stifterverband für die Deutsche Wissenschaft für ihre Unterstützung.

Ein Archiv der Polen-Analysen finden Sie im Internet unter w w w.laender-analysen.de/ polen

Für ein kostenloses Abonnement senden Sie eine E-Mail mit dem Stichwort PolenAnalysen in der Betreffzeile an polen-analysen@dpi-da.de

\title{
Deutsches Polen-Institut Darmstadt
}

Das Deutsche Polen-Institut Darmstadt (DPI) ist ein Forschungs-, Informations-, und Veranstaltungszentrum für polnische Kultur, Geschichte, Politik, Gesellschaft und die deutsch-polnischen Beziehungen, die sich im Kontext der europäischen Integration entwickeln. Das seit März 1980 aktive und bis 1997 von Gründungsdirektor Karl Dedecius geleitete Institut ist eine Gemeinschaftsgründung der Stadt Darmstadt, der Länder Hessen und Rheinland-Pfalz sowie des Bundes. Seit 1987 ist die Trägerschaft auf die Kultusministerkonferenz der Länder ausgedehnt. Einen wesentlichen Beitrag zur Verwirklichung der Institutsziele leisten private Stiftungen. Das DPI hat satzungsgemäß die Aufgabe, durch seine Arbeit zur Vertiefung der gegenseitigen Kenntnisse des kulturellen, geistigen und gesellschaftlichen Lebens von Polen und Deutschen beizutragen.

Ziel der Vermittlertätigkeit des DPI ist es, "die zu interessieren, auf die es politisch, wirtschaftlich, gesellschaftlich und kulturell im deutsch-polnischen Verhältnis ankommt (Leitlinien 1997). Es geht um die Entscheider und Multiplikatoren in Politik, Kultur, Bildung, Verwaltung, Medien und Wirtschaft und, wesentlich stärker ausgeprägt als bisher, um das Hineinwirken in Wissenschaft, Forschung und Bildung.

Derzeit bemüht sich das DPI in Kooperation mit den verstreuten Orten wissenschaftlicher Polen-Kompetenz an deutschen Hochschulen und Forschungsinstituten verstärkt darum, ausgehend von einer Bestandsaufnahme deutscher Polen-Forschung Ort wissenschaftlicher Forschung und verbindendes, vernetzendes und kooperierendes Zentrum zu werden. Ausgangspunkt der Neuausrichtung ist die kaum mehr kontrollierbare Dynamik des Rückbaus der Ressourcen der wissenschaftlichen Polen-Kompetenz in den unterschiedlichen Disziplinen. Mit der über 50.000 Bände zählenden multidisziplinären Fachbibliothek für Polen mit einer einzigartigen Sammlung polnischer Literatur in der Originalsprache und in deutscher Übersetzung ist das DPI bereits ein geschätzter Ort der Recherche und des wissenschaftlichen Arbeitens. (w w w.deutsches-polen-institutde)

\section{Forschungsstelle Osteuropa an der Universität Bremen}

1982 gegründet, widmet sich die Forschungsstelle Osteuropa an der Universität Bremen unter der Leitung von Prof. Dr. Eichwede kulturellen und gesellschaftlichen Entwicklungen der Länder Ost- und Ostmitteleuropas in Zeitgeschichte und Gegenwart. Die Forschungsstelle besitzt in ihrem Archiv eine einzigartige Sammlung alternativer Kulturgüter und unabhängiger Texte aus den ehemaligen sozialistischen Ländern. Darunter befindet sich auch eine umfangreiche Sammlung des "Zweiten Umlaufs«, die das Schrifttum und Dokumente unabhängiger Initiativen und gesellschaftlicher Gruppen in Polen aus der Zeit von 1976 bis zum Umbruch umfasst. Neben ausführlicher individueller Forschung zu Dissens und Gesellschaft im Sozialismus, leitet die Forschungsstelle seit Januar 2007 ein gemeinsames Projekt mit einem Verbund von internationalen Forschungsinstituten zum Thema »Das andere Osteuropa - die 1960 er bis 1980er Jahre, Dissens in Politik und Gesellschaft, Alternativen in der Kultur. Beiträge zu einer vergleichenden Zeitgeschichte«, welches von der VolkswagenStiftung finanziert wird.

Im Bereich der post-sozialistischen Gesellschaften sind in den letzten Jahren umfangreiche Forschungsprojekte durchgeführt worden, deren Schwerpunkte auf politischen Entscheidungsprozessen, Wirtschaftskultur und der EU-Osterweiterung lagen. Eine der Hauptaufgaben der Forschungsstelle ist die Information der interessierten Öffentlichkeit. Dazu gehören unter anderem regelmäßige E-Mail-Informationsdienste mit fast 15.000 Abonnenten in Politik, Wirtschaft und den Medien.

Mit ihrer in Deutschland einzigartigen Sammlung von Publikationen zu Osteuropa ist die Forschungsstelle eine Anlaufstelle sowohl für Wissenschaftler als auch für die interessierte Öffentlichkeit. In der Bibliothek sind derzeit neben anderen breit angelegten Beständen allein aus Polen ca. 300 laufende Periodika zugänglich. Die Bestände werden in Datenbanken systematisch erfasst. (w w w.fo rschung sstelle.uni-b remen.de)

\author{
Die Meinungen, die in den Polen-Analysen geäußert werden, geben ausschließlich die Auffassung der Autoren wieder. \\ Abdruck und sonstige publizistische Nutzung sind nach Rücksprache mit der Redaktion gestattet. \\ Redaktion: Prof. Dr. Dieter Bingen (Darmstadt), Dr. Stefan Garsztecki (Bremen), Silke Plate, M.A. (Bremen) \\ Technische Gestaltung: Matthias Neumann \\ Polen-Analysen-Layout: Cengiz Kibaroglu, Matthias Neumann \\ ISSN 1863-9712 (C) 2008 by Deutsches Polen-Institut Darmstadt und Forschungsstelle Osteuropa, Bremen \\ Kontakt: Dr. Andrzej Kaluza, Presse- und Öffentlichkeitsarbeit, Deutsches Polen-Institut, Mathildenhöhweg 2, \\ D-64287 Darmstadt, Tel.: 06151/4985-13, Fax: 06151/4985-10, E-Mail: polen-analysen@dpi-da.de, Internet: www.laender-analysen.de/polen
}

